\title{
CRIPTOCOCOSE EM FELINO: relato de caso
}

\section{Cryptococcosis in a feline - case report}

\author{
Talita Oliveira Rodrigues ${ }^{\star 1}$; juliana Reis Godoy ${ }^{1}$; Patrícia Malandrim ${ }^{1}$; \\ Vinícius Sossai ${ }^{1}$; Mariely Thaís De Souza ${ }^{2}$ \\ *Autor Correspondente: Talita Oliveira Rodrigues, Rua Olímpia de Almeida Prado, 27, Apto 402, \\ Barra Funda, São Paulo, SP - Cep 01151-010. \\ E-mail: mv.oliveiratalita@gmail.com.
}

\begin{abstract}
Como citar: RODRIGUES, Talita Oliveira; GODOY, Juliana Reis; MALANDRIM, Patrícia; SOSSAI, Vinícius; DE SOUZA, Mariely Thaís. Criptococose em felino: relato de caso. Revista de Educação Continuada em Medicina

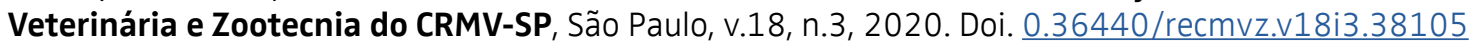

Cite as: RODRIGUES, Talita Oliveira; GODOY, Juliana Reis; MALANDRIM, Patrícia; SOSSAI, Vinícius; DE SOUZA, Mariely Thaís. Cryptococcosis in feline: case report. Journal of Continuing Education in Animal Science of CRMV-SP, São Paulo, v.18, n.3, 2020. Doi. $0.36440 /$ recmvz.v18i3.38105
\end{abstract}

\section{Resumo}

0 presente trabalho analisa um caso de criptococose em um felino doméstico, sem raça definida, com onze anos de idade, atendido no Hospital Veterinário "Dr. Vicente Borelli", do Centro Universitário Fundação de Ensino Octávio Bastos, em São João da Boa Vista - São Paulo. 0 animal apresentava aumento de volume em região nasal. Após exame clínico, foi realizada a colheita de material para exame de cultura fúngica e histopatológico. Neste último, observou-se uma dermatite piogranulomatosa e presença do microrganismo Cryptococcus spp., capaz de acometer humanos e animais domésticos e silvestres, confirmando o diagnóstico de Criptococose.

Palavras-chave: Criptococose. Felino. Microrganismos. Diagnóstico. Terapêutica.

\section{Summary}

This paper analyzes a case report of cryptococcosis in a 11 years old crossbreed domestic feline, that was attended at "Dr. Vicente Borelli" Centro Universitário Fundação de Ensino Octávio Bastos Veterinary Hospital. The animal presented increased nasal volume. After physical examination, it was collected material from the lesion in order to do fungal culture and histologic analysis. It was observed pyogranulomatous dermatitis and presence of Cryptococcus spp., an microorganism able to affect humans, domestics and wild animals, corroborating the diagnosis of Cryptococcosis.

Keywords: Cryptococcosis. Feline. Microorganism. Diagnosis. Therapy.

1 Aprimorando (a) em Clínica Médica e Cirúrgica de Pequenos Animais, Hospital Veterinário "Dr. Vicente Borelli", Faculdade Pio Décimo, Campus Jabutiana, Jabutiana, Aracaju, SE, Brasil

2 Docente do curso de Medicina Veterinária, Centro Universitário, Fundação de Ensino Octávio Bastos, São João da Boa Vista, SP, Brasil 


\section{Introdução}

A criptococose, também conhecida como Torulose, Blastomicose Europeia e Doença de Busse-Buschke, é uma micose cosmopolita, de natureza sistêmica oportunista, de caráter zoonótico (MÜLLER; NISHIZAWA, 2017) e distribuição mundial (MCGILL et al., 2009; PIMENTA et al., 2015; BRITO-SANTOS et al., 2019). No Brasil, foi descrita a primeira vez em um gato doméstico em 1971 (OLIVEIRA; NOBRE; FERREIRO, 2005; FARIA, 2015). Contudo, poucos casos foram documentados desde então (BRITO-SANTOS et al., 2019).

Quanto à transmissão, a infecção ocorre por inalação de esporos no ambiente, os quais se desenvolvem em matérias orgânicas, tais como excrementos de aves, morcegos e, principalmente, pombos urbanos, devido ao alto teor de nitrogênio e creatinina fornecido por esse substrato, favorecendo maior crescimento do agente (PINTO FILHO et al., 2003; MCGILL et al., 2009; FARIA, 2015; MÜLLER; NISHIZAWA, 2017), além de eucalipto em decomposição (RONDELLI et al., 2010; PIMENTA et al., 2015). Mais raramente pode haver inoculação cutânea direta (MCGILL et al., 2009). Trata-se, portanto, de uma saprozoonose (LAPPIN, 2015; MÜLLER; NISHIZAWA, 2017). A doença infecciosa é causada por leveduras saprófitas, com células envoltas por uma cápsula polissacarídica, pertencentes ao gênero Cryptococcus, sendo as duas espécies patogênicas $C$. neoformans e $C$. gattii (PERFECT; BICANIC, 2015) outras espécies raramente foram relatadas como causadoras da doença (PINTO FILHO et al., 2003; FARIA, 2015).

A enfermidade fúngica em questão está mais associada a humanos e animais de companhia, em especial, aos gatos (RONDELLI et al., 2010; FARIA, 2015; PIMENTA et al., 2015), aos animais de produção e, mais raramente, aos animais silvestres (MÜLLER; NISHIZAWA, 2017). A maior ocorrência da doença em gatos é provavelmente ocasionada devido ao seu comportamento de limpeza, o que aumenta a probabilidade de acesso dos fungos à cavidade nasal (PIMENTA et al., 2015). Tornase mais grave com condições imunossupressoras preexistentes, como em animais em tratamento prolongado com corticosteroides, com quimioterápicos, ou portadores de doenças como as síndromes da imunodeficiência felina (FIV) e leucemia felina (FeLV) nos gatos e, nos cães, erliquiose e dirofilariose (LAPPIN, 2015). Gatos de vida livre rural parecem estar mais expostos ao Cryptococcus (PIMENTA et al., 2015).

Existe uma variabilidade na patogênese entre as diferentes espécies, que pode estar relacionada aos diferentes tamanhos das estruturas das conchas nasais, no comprimento da traqueia, na eficiência dos mecanismos de depuração mucociliar e reflexo da tosse, ou exposição a diferentes tipos de propágulos infecciosos (MCGILL et al.., 2009).

O período de incubação varia de meses a anos (PIMENTA et al., 2015). Os seus sinais são divididos em quatro síndromes, que podem aparecer de forma isolada ou conjunta: síndrome respiratória, neurológica, ocular e cutânea (QUEIROZ et al., 2008; MÜLLER; NISHIZAWA, 2017). A evolução do quadro está correlacionada a resposta imunológica do paciente no momento da infecção (QUEIROZ et al., 2008).

A síndrome respiratória, mais frequente no gato (MCGILL et al., 2009; MÜLLER; NISHIZAWA, 2017), afeta com maior frequência a cavidade nasal (PIMENTA et al., 2015) do que pulmões (HONSHO et al., 2003), é caracterizada por roncos, estertores, corrimento nasal, uni ou bilateral, mucopurulento, seroso ou sanguinolento, espirros e dispneia inspiratória. Pode ocorrer a formação de massas firmes ou pólipos no tecido subcutâneo, especialmente sobre a cartilagem do plano nasal, levando ao aumento na narina e vermelhidão, popularmente conhecido como "nariz de palhaço" (FARIA, 2015; LAPPIN, 2015; MÜLLER; NISHIZAWA, 2017). Contudo, pode haver também infecção pulmonar, e os microrganismos, após se disseminarem a níveis alveolares, ganham a via hematógena e atingem diversos órgãos, como os rins, ocasionando insuficiência renal secundária, ou claudicação secundária a osteomielite e difundida linfadenopatia (RONDELLI et al., 2010; FARIA, 2015; LAPPIN, 2015).

Os animais acometidos pela síndrome cutânea podem apresentar nódulos múltiplos na pele (RONDELLI et al., 2010) ou subcutâneo, preferencialmente na cabeça ou pescoço dos gatos, de 
crescimento rápido (MÜLLER; NISHIZAWA, 2017), que podem ser firmes ou flutuantes, ulcerar e drenar um exsudato serosanguinolento (LAPPIN, 2015; MÜLLER; NISHIZAWA, 2017); as lesões podem ocorrer em nariz, língua, gengivas, palato duro, lábios ou leito ungueal (FARIA, 2015).

Quando há disseminação da criptococose a nível ocular, manifesta-se um complexo de sinais incluindo fotofobia, blefaroespasmo, opacidade da córnea, edema inflamatório da íris, hifema (MÜLLER; NISHIZAWA, 2017), coriorretinite granulomatosa, blefaroespasmo (HONSHO et al., 2003), uveíte anterior, neurite óptica, luxação das lentes, descolamento retinal supurativo e glaucoma (LAPPIN, 2015).

Os sinais nervosos, mais comuns em cães (MCGILL et al., 2009), são resultantes da meningoencefalite difusa ou focal da formação granulomatosa (LAPPIN, 2015). Podem estar incluídas nas manifestações clínicas movimentos em círculos, depressão, mudanças de comportamentos, angústia, cegueira, perda de olfato, ataxia, tetraparesia, paraplegia, além de doença vestibular periférica (FARIA, 2015; LAPPIN, 2015), hiperestesia cervical, anisocoria, midríase, surdez, convulsões (MÜLLER; NISHIZAWA, 2017), inclinação da cabeça (head tilt) e nistagmo (RONDELLI et al., 2010).

O diagnóstico presuntivo da criptococose é construído a partir da anamnese, dados epidemiológicos, sinais clínicos e patológicos (FARIA, 2015; PERFECT; BICANIC, 2015). 0 diagnóstico definitivo é obtido com a demonstração da levedura no material clínico, por exame direto com tinta da China (nanquim) (FARIA, 2015; MÜLLER.; NISHIZAWA, 2017; PERFECT; BICANIC, 2015;), cultura fúngica, exame histopatológico e pesquisa de antígenos circulantes (FARIA, 2015; LAPPIN, 2015; PERFECT; BICANIC, 2015; PIMENTA et al., 2015).

0 exame micológico comprobatório da doença, pode ser realizado em swabs de exudatos, (JULIANO; SOUZA; SCHEIDE, 2006; FARIA, 2015), líquor, lavado broncoalveolar, fragmentos de biópsia (FARIA, 2015; LAPPIN, 2015), aspirado de medula óssea, linfonodos e urina (MÜLLER; NISHIZAWA, 2017).

Podem ser utilizados corantes como ácido periódico-Shiff (PAS), hematoxifilina-eosina (H\&E) e Grocott-Gomori no exame histopatológico, em que são visualizadas células leveduriformes de tamanho distinto, com ou sem brotamento, com um espaço circular claro correspondente à cápsula, e inflamação piogranulomatosa e granulomatosa. 0 material pode ainda ser corado pelo mucicarmim de Mayer, que cora a cápsula do Cryptococcus de rosa-avermelhado, possibilitando a diferenciação entre outras leveduras não capsuladas (QUEIROZ et al., 2008; FARIA, 2015).

0 cultivo deve ser efetuado em meios específicos e incubado a temperaturas em torno de 30 a $35^{\circ} \mathrm{C}$, as colônias podem ser observadas após 48 a $72 \mathrm{~h}$, fazendo-se a análise microscópica delas e provas bioquímicas para confirmação do agente (QUEIROZ et al., 2008; FARIA, 2015; LAPPIN, 2015).

Os testes sorológicos mais empregados para o diagnóstico da criptococose são aglutinação em látex e ELISA, com altas taxas de sensibilidade e especificidade, eventualmente surgindo casos de falsopositivos (LAPPIN, 2015; PERFECT; BICANIC, 2015), e falso-negativos, nas infecções precoces (PERFECT; BICANIC, 2015).

Pinto Filho et al. (2003) e Pimenta et al. (2015) destacam que o tratamento da criptococose deverá ter continuidade por um período mínimo de dois meses após a resolução dos sinais clínicos da doença. Faria (2015) aponta que a duração do tratamento deve basear-se na resposta do paciente e resultados negativos de acompanhamento da citologia ou cultura. Além do mais, a medicação contínua para os animais de estimação deve ser administrada por período prolongado (em média seis a dezoito meses), o paciente deve ter acompanhamento clínico até que se tenha eliminado completamente 0 agente do organismo, é necessário estender o tratamento por pelo menos um a dois meses após cura clínica, para que não ocorra a recidiva das lesões.

São recomendados no tratamento da criptococose antifúngicos sistêmicos como anfotericina B, cetoconazol, itraconazol, voriconazol ou 5-fluocitosina (RONDELLI et al., 2010; LAPPPIN, 2015). 0 itraconazol é o antifúngico de escolha para cães e gatos quando não há envolvimento do SNC, é 
mais seguro e eficaz que o cetoconazol quanto a efeitos colaterais e duração de tratamento, além de apresentar menor custo que o fluconazol. É administrado em cães e gatos na dose de $10 \mathrm{mg} / \mathrm{kg}$, uma vez ao dia, pela via oral (FARIA, 2015; PINTO FILHO et al., 2003). Sousa (2015) aponta a dose do itraconazol de $10-15 \mathrm{mg} / \mathrm{kg}$, via oral, uma vez ao dia e com uma dieta rica em gordura.

A prevenção da criptococose é obtida com a redução do potencial de exposição, ou seja, as áreas com altas concentrações de excretas de aves devem ser evitadas (FARIA, 2015), visto que o fungo eliminado nas fezes de aves, principalmente pombos, pode permanecer viável e infectante por mais de dois anos (MÜLLER; NISHIZAWA, 2017). Além disso, Faria (2015) aponta que a limpeza periódica de locais com potencial para contaminação pelo agente da criptococose deve ser úmida, para evitar aerossolização da poeira contaminada, com solução de formalina ou formaldeído a 3\% e proteção com máscaras e luvas. Pinto Filho (2003) ressalta que, nas áreas de risco deve ser efetuado o tratamento dos excrementos das aves com soda cáustica ou cal. Lappin (2015) preconiza a aplicação de lima hidratada para reduzir o número de organismos em áreas contaminadas.

O prognóstico da criptococose vai depender do estado do paciente, da presença ou não de sinais neurológicos, da existência de doenças imunossupressoras e da cooperação do tutor, já que o tratamento é longo e de custo relativamente elevado (FARIA, 2015). Com relação à saúde pública, a criptococose não constitui uma antropozoonose clássica, pois o agente não sofre aerossolização a partir de tecidos ou secreções contaminadas (FARIA, 2015; PINTO FILHO, 2003). Entretanto, seu surgimento em centros urbanos é um desafio a indivíduos e animais imunocompetentes (BRITO-SANTOS et al., 2019) e, especialmente, aos imunossuprimidos, pois para estes, as consequências são graves e potencialmente fatais (MÜLLER; NISHIZAWA, 2017).

\section{Relato do caso}

Foi atendido no Hospital Veterinário "Dr. Vicente Borelli" do Centro Universitário Fundação de Ensino Octávio Bastos, São João da Boa Vista - SP, um gato, sem raça definida, macho, 4,9kg, com 11 anos de idade e histórico de aumento de volume nodular em região nasal. A formação havia surgido há sete dias, e o maior crescimento havia sido constatado nos dois dias que antecederam da consulta. O animal não apresentava nenhuma alteração além da descrita. Entretanto, possuía vida rural livre e tinha acesso a aves, incluindo pombos.

Figura 1. A. Animal apresentando massa em região nasal ("nariz de palhaço") na primeira consulta. B. Lesão após 35 dias de tratamento. C. Lesão após 60 dias de tratamento
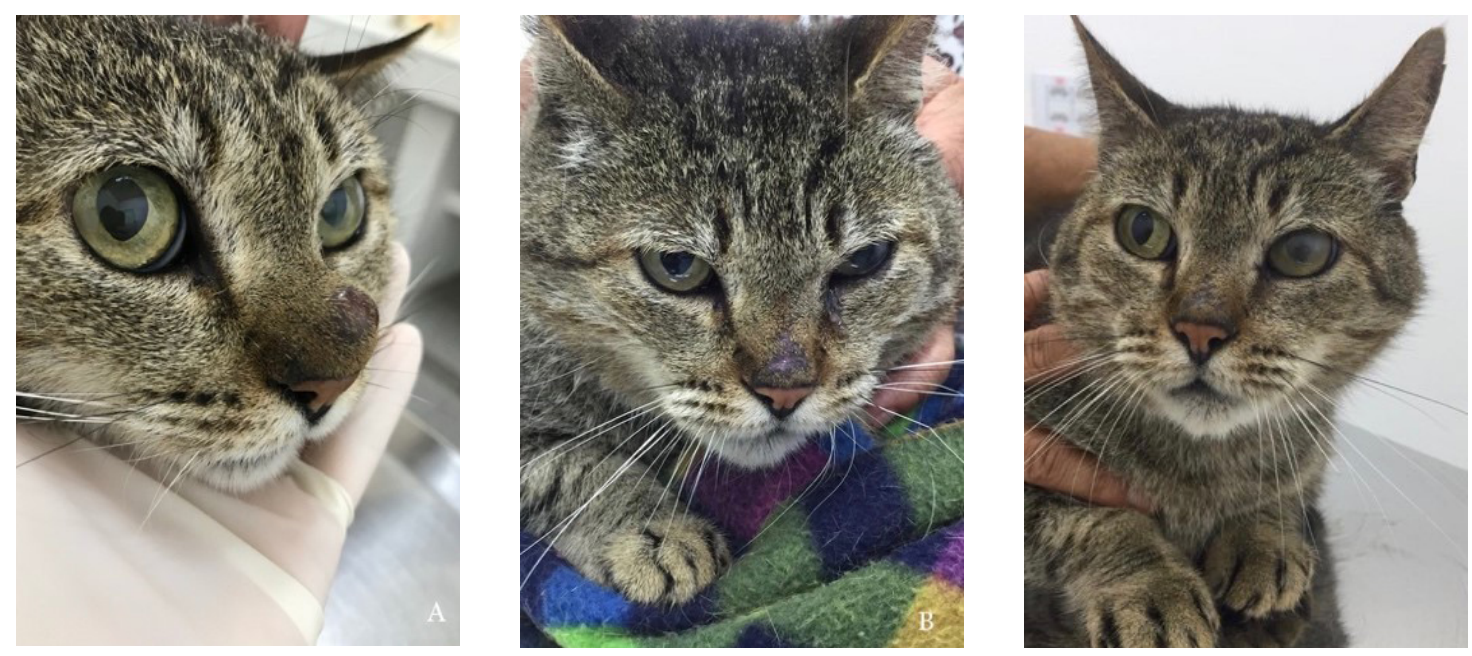

T Fonte: Rodrigues (2018). 
0 exame físico revelou deformidade nasal de consistência firme, sem sensibilidade à dor e não ulcerada. A temperatura retal era de $38,3^{\circ} \mathrm{C}$, frequência cardíaca de 186 batimentos por minuto e frequência respiratória de 44 movimentos por minuto. Não foi observada qualquer anormalidade na auscultação pulmonar ou nos demais parâmetros fisiológicos.

Os exames laboratoriais revelaram trombocitopenia e discreta hiperproteinemia. Não houve alteração na bioquímica sérica.

0 paciente foi anestesiado e a seguir foi realizada a colheita de material para exame de cultura fúngica em meios específicos e análise histopatológica.

O diagnóstico definitivo foi obtido por meio do exame histopatológico, utilizando o método de coloração com hematoxilina-eosina, que evidenciou intenso infiltrado inflamatório em derme, composto por neutrófilos e macrófagos associados a raras formas fúngicas de Cryptococcus spp centrais, além de focos de necrose. Quadro de dermatite piogranulomatosa compatível com criptococose. 0 exame de cultura fúngica foi negativo para dermatófitos, sendo isolado um microrganismo denominado Acremonium spp.

Após o procedimento de biópsia e antes do resultado do histopatológico, foi iniciado o tratamento com itraconazol $10 \mathrm{mg} / \mathrm{kg}$, BID, durante pelo menos noventa dias; óleo de peixe (ômega 3), $1 \mathrm{~g} / 5 \mathrm{~kg}$, $\mathrm{SID}$, durante pelo menos noventa dias; cefalexina $30 \mathrm{mg} / \mathrm{kg}$, BID, dez dias; precedido de cloridrato de ranitidina $2 \mathrm{mg} / \mathrm{kg}$, BID, durante dez dias; dipirona $25 \mathrm{mg} / \mathrm{kg}$, SID, durante cinco dias.

Houve acompanhamento do animal por um pouco mais de dois meses seguintes ao tratamento, e o mesmo não apresentou sinais de recidiva. Contudo, ainda que a importância dessa saprozoonose, as informações e riscos tenham sido informados à tutora, a mesma não retornou com os 90 dias de tratamento conforme orientação médico-veterinária. Durante o tempo de acompanhamento, nenhum sinal clínico havia sido observado na tutora ou nos contactantes do paciente, contudo ela foi orientada a procurar um médico caso apresentasse algum sintoma.

\section{Discussão}

A disseminação mundial do Cryptococcus spp (BRITO-SANTOS et al., 2019) culmina em grande relevância para a criptococose tanto para animais, quanto para humanos, não somente aos indivíduos imunossuprimidos, cujas consequências são graves e potencialmente fatais (MÜLLER; NISHIZAWA, 2017), mas, de forma geral, também aos imunocompetentes (BRITO-SANTOS et al., 2019).

0 contato com pombos ocorre em diversos locais, como em praças, parques e nas residências, o que pode ocasionar o acesso às excretas destas aves, que são ricas em compostos nitrogenados, e facilitam o desenvolvimento do agente da criptococose (PINTO FILHO et al., 2003; FARIA, 2015; MÜLLER; NISHIZAWA, 2017). Além do mais, gatos de vida rural livre, como o animal do presente relato, parecem estar mais expostos à infecção (PIMENTA et al., 2015).

As lesões características de criptococose em felinos têm sido descritas por inúmeros autores, e o exame clínico do caso em questão revelou padrões lesionais bem compatíveis com os descritos por Lappin (2015) e Faria (2015).

O diagnóstico do presente caso foi obtido por meio de análise histopatológica. Contudo, esta é uma técnica que requer conhecimento do laboratorista, pois o C. neoformans é encontrado nas amostras, sob a forma de levedura oval e arredondada, que, por sua vez, quando presente, não se cora por qualquer corante devido a cápsula lipídica, fazendo-se necessário o uso de corantes como PAS, H\&E, Grocott-Gomori e mucicarmim de Mayer (FARIA, 2015; QUEIROZ et al. 2008), no presente relato, a coloração utilizada foi H\&E.

Lappin (2015) destacou que a criptococose tende a provocar infecção mais grave em animais imunossuprimidos, entretanto, no paciente do presente relato, por questões financeiras, não foram realizados os testes para as enfermidades FIV e FeLV. 
Após o estabelecimento do diagnóstico de criptococose foi prescrito itraconazol, devido ao menor grau de toxicidade quando comparado ao cetoconazol (LAPPIN, 2015), e também por ser uma das drogas de eleição para o tratamento da enfermidade quando não há envolvimento do SNC, além de óleo de peixe, uma fonte de gordura, como descrito em Silva (2015).

Embora Pinto Filho et al. (2003) e Pimenta et al. (2015) apontem que o tratamento da criptococose deve ter continuidade por um período mínimo de dois meses após a resolução dos sinais clínicos da doença, como a tutora não retornou com os 90 dias de tratamento como orientado, não foi possível o acompanhamento dos sinais a partir desse momento, ou realizar os exames de comprovação de negativação da enfermidade. Faria (2015) aponta um custo elevado para o tratamento da enfermidade, o que pode ter influenciado o comportamento da tutora do animal descrito no presente relato de caso.

A tutora do animal acometido pela criptococose foi alertada sobre a importância e os riscos da micose acometer seres humanos, e da necessidade da procura por um médico para a obtenção de maiores informações. Durante os meses de acompanhamento, não foi verificada a presença de sintomatologia na mesma ou em outras pessoas do convívio do paciente, reafirmando que os animais infectados não são importantes na transmissão entre outros animais e pessoas, pois trata-se de uma saprozoonose, como apontado em 2017 por Müller e Nishizawa.

\section{Conclusões}

Embora não seja uma antropozoonose clássica, a criptococose é um desafio de relevância à saúde pública devido a sua ampla distribuição geográfica. É uma enfermidade de grande importância em animais de companhia, especialmente, nos felinos, sendo considerada a micose sistêmica que mais acomete estes últimos.

Juntamente com a anamnese, dados epidemiológicos e sinais clínicos da doença, embora muitas vezes inespecíficos, é importante a tomada de decisões para escolha dos exames comprobatórios da enfermidade e posterior tratamento, preferencialmente de forma precoce. 0 prognóstico é reservado e depende do estado clínico do animal, da presença de doenças concomitantes e da utilização de drogas imunossupressoras. 


\section{Referências}

BRITO-SANTOS, F. et al. Cryptococcosis due to Cryptococcus gattii VGII in southeast Brazil: The One Health approach revealing a possible role for domestic cats. Elsevier, Rio de Janeiro, v. 24, p. 61-64, jun. 2019. Disponível em: https://doi.org/10.1016/j.mmcr.2019.04.004. Acesso em: 09 de dez. 2020.

FARIA, R. O. Fungos dimórficos e relacionados com micoses profundas. In: GERICÓ, M. M.; ANDRADE NETO, J. P.; KOGIKA, M. M. (Org.). Tratado de medicina interna de cães e gatos. Rio de Janeiro: Roca, p. 2395-2419, 2015.

HONSHO, C. S. et al. Generalized systemic cryptococcosis in a dog after immunosuppressive corticotherapy. Arquivo Brasileiro de Medicina Veterinária e Zootecnia, v. 55, p. 155-159, 2003.

JULIANO, R. S.; SOUZA, A. I.; SCHEIDE, R. Criptococose felina. Revista de Patologia Tropical, v. 35, n.1, p.65-70, 2006. Disponível em: https://doi.org/10.5216/rpt.v35i1.1895. Acesso em: 09 de dez. 2020.

LAPPIN, M. R. Criptococose. In: NELSON, R. W.; COUTO, C. G. (Ed.). Medicina interna de pequenos animais. 5. ed. Rio de Janeiro: Elsevier, p. 1360-1363, 2015.

MCGILL, S. et al. Cryptococcosis in domestic animals in Western Australia: a retrospective study from 1995-2006. Medical Mycology, v. 47, n. 6, p. 625-639, 2009. Disponível em: https://doi.org/10.1080/13693780802512519. Acesso em: 09 de dez. 2020.

MÜLLER, M.; NISHIZAWA, M. A criptococose e sua importância na Medicina Veterinária. Revista de Educação Continuada em Medicina Veterinária e Zootecnia do CRMV-SP, v. 15, n. 1, p. 24-29, 2017. Disponível em: https://doi.org/10.36440/recmvz.v15i1.36761. Acesso em: 09 de dez. 2020.

QUEIROZ, J. P. A. F. et al. Criptococose: uma revisão bibliográfica. Acta Veterinaria Brasilica, v. 2, n. 2, p. 32-28, 2008. Disponível em: https://doi.org/10.21708/avb.2008.2.2.699. Acesso em: 09 de dez. 2020.

OLIVEIRA, I. A.; NOBRE, M. O.; FERREIRO, L. Pesquisa de criptococose em cães atendidos no Hospital das Clínicas Veterinárias da UFRGS, Porto Alegre, Brasil. Acta Scientiae, v. 33, n. 3, p. 253-258, 2005. Disponível em: https://doi.org/10.22456/1679-9216.14945. Acesso em: 09 de dez. 2020.

PERFECT, J. R.; BICANIC, T. Cryptococcosis diagnosis and treatment: What do we know now. Fungal Genetics and Biology, Durham, v. 78, p. 49-54, 2015. Disponível em: https://doi.org/10.1016/j. fgb.2014.10.003. Acesso em: 09 de dez. 2020.

PIMENTA, P. et al. Blepharitis due to Cryptococcus neoformans in a cat from northen Portugal. Journal of Feline Medicine and Surgery Open Reports, v. 1, n. 2, 2015. Disponivel em: https://journals.sagepub. com/doi/10.1177/2055116915593963. Acesso em: 16 nov. 2019.

PINTO FILHO, S. T. L. et al. Criptococose nasal e cutânea em felino: relato de caso. MEDVEP. Rev. Científica de Medicina Veterinária de Pequenos Animais e Animais de Estimação, v. 2, n. 5, p. 33-37, 2003.

RONDELLI, M. C. H et al. E. Criptococose diagnosticada por imprints de lesões em mucosa oral em cão. Arquivo Brasileiro de Medicina Veterinária e Zootecnia, v. 62, n. 5, p. 1271-1274, 2010. Disponível em: https://doi.org/10.1590/S0102-09352010000500035. Acesso em: 09 de dez. 2020.

SOUSA, M. G. Doenças infecciosas. In: CRIVELLENTI, L. Z.; BORIN-CRIVELLENTI, S. (Org.) Casos de rotina em Medicina Veterinária de pequenos animais. São Paulo: Editora MedVet, p. 156-157, 2015. 